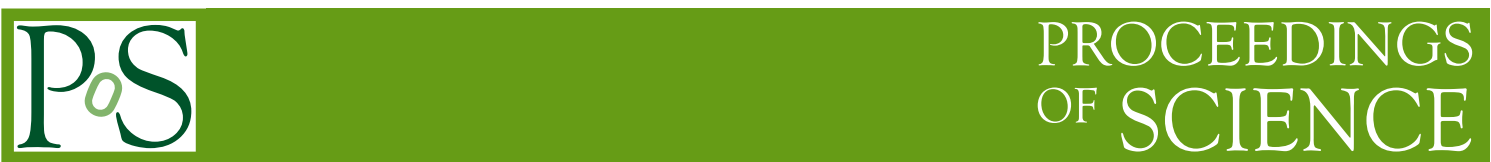

\title{
Upgrade of MEG Liquid Xenon Calorimeter
}

\section{Ryu SAWADA*}

$\dagger$

ICEPP, the University of Tokyo

E-mail: sawada@icepp.s.u-tokyo.ac.jp

The MEG experiment yielded the most stringent upper limit on the branching ratio of the flavorviolating muon decay $\mu^{+} \rightarrow e^{+} \gamma$. A major upgrade of the detector is planned to improve the sensitivity by one order of magnitude. For the upgrade of the liquid xenon (LXe) scintillation gamma detector, 2-inch round-shape photomultiplier tubes (PMTs) on the inner face of the detector will be replaced by $12 \times 12 \mathrm{~mm}^{2}$ Multi-Pixel Photon Counters (MPPCs) to significantly improve the granularity. The higher granularity will improve the energy resolution from $2.4 \%$ to $1.1 \%$ and the position resolution from $5 \mathrm{~mm}$ to $2 \mathrm{~mm}$ near the inner face. The MPPC in the upgraded LXe detector is required to have a high photon detection efficiency (PDE) for the LXe scintillation light in the VUV range with a good gain uniformity and to be operational in the LXe temperature $(165 \mathrm{~K})$. Ultraviolet-enhanced MPPCs are being developed in collaboration with Hamamatsu Photonics and were tested in LXe. The single-photoelectron detection capability was confirmed, and the PDE for the LXe scintillation light was measured to be $\sim 15 \%$. The design and the expected performance of the upgraded LXe detector and the status of the detector developments are presented.

Technology and Instrumentation in Particle Physics 2014

2-6 June, 2014

Amsterdam, the Netherlands

* Speaker.

${ }^{\dagger}$ on behalf of the MEG II collaboration 


\section{MEG experiment and upgrade}

The MEG experiment is carried out to search for the charge-lepton flavor violating muon decay $\mu^{+} \rightarrow e^{+} \gamma$. In the standard model of the particle physics, flavor violating processes in the charged sector (CLFV) are forbidden; however such processes are predicted to happen with observable branching ratios in many new theories beyond the standard model. A discovery of CLFV decays would be therefore an unambiguous evidence of new physics. The signature of the $\mu^{+} \rightarrow e^{+} \gamma$ decay of stopped muons is pairs of gamma ray and positron emitted to the opposite directions with monochromatic energy of half the muon mass. The dominant background is the accidental coincidence of positrons from the normal muon decays and gamma rays from either the radiative muon decay or the annihilation of positrons hitting detector materials. For suppressing the background rate, the positron detector have to be made of light materials, and the detector has to have excellent energy, angle and time resolutions. The MEG experiment took data in year 20092013 at Paul Scherrer Institute (PSI) where the most intense DC muon beam is available. In year 2013, we published the latest result based on the analysis of data taken in year 2009-2011 [1]. In the analysis, no evidence of the signal was found and the upper limit of the branching ratio was set to be $5.7 \times 10^{-13}$. In parallel to the analysis of the remaining data, the collaboration started the upgrade of the experiment to achieve ten times better sensitivity for the signal. The proposal was approved by the PSI committee in year 2013, and the development of the new detectors is ongoing.

\section{MEG liquid xenon calorimeter}

The gamma-ray detector for the MEG experiment is a liquid xenon (LXe) scintillation detector. In 900 liters of liquefied xenon, 846 photomultiplier tubes (PMT) are submerged for directly detecting the scintillation light from the LXe (Fig. 1). There are several advantages to use LXe as a scintillation medium such as,

- High scintillation efficiency close to NaI,

- Fast fall time of the scintillation signal ( $\lambda=45 \mathrm{nsec}$ for gamma rays),

- High stopping power,

- Homogeneous response over the large detection volume.

On the other hand, the peak wavelength of the scintillation light is about $175 \mathrm{~nm}$ for which normal PMTs do not have sensitivity. We therefore developed a new type of PMTs, which has sensitivity for vacuum ultraviolet (VUV) light, with Hamamatsu Photonics. The 2-inch PMTs developed for the MEG experiment (R9869) have the product of the quantum efficiency and the collection efficiency of typically $16 \%$ for the VUV light. They can be used in the LXe temperature $(165 \mathrm{~K})$ without contaminating LXe.

\subsection{Present detector and resolutions}

Table 1 shows the performance of the present MEG gamma-ray detector. The energy and position resolutions depend on the conversion depth because the photon collection efficiency in the 

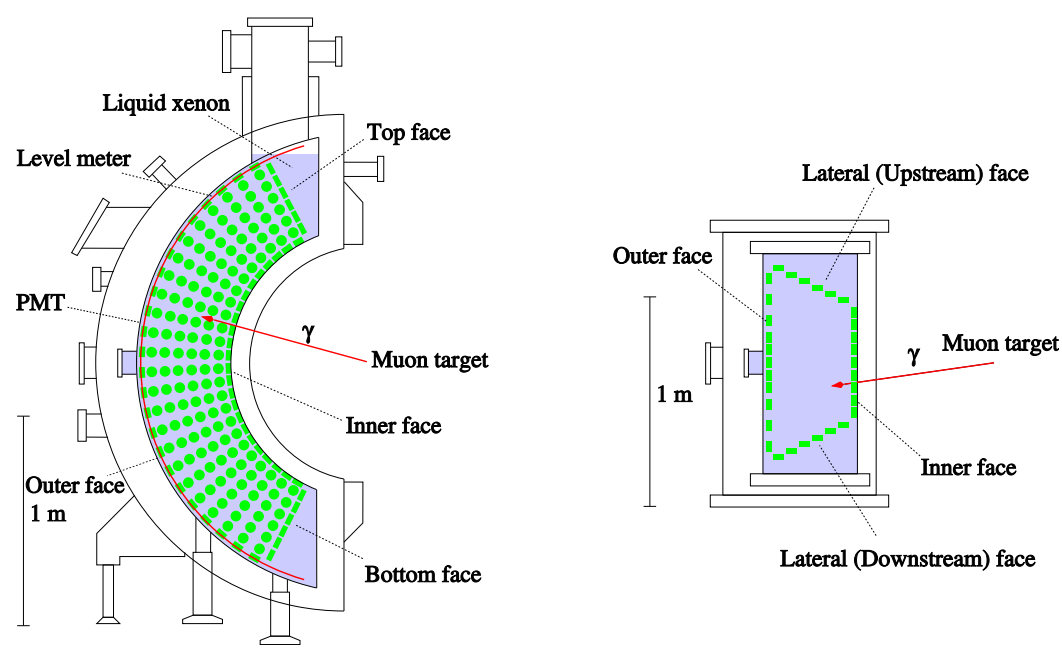

Lateral (Downstream) face

Figure 1: MEG LXe gamma ray detector. Gamma rays from the muon-stopping target enter the liquid xenon volume through the inner face and are converted near the face.

detector strongly depends on the incidence position relative to the PMT array on the inner face (Fig. 2). Because of the short radiation length of LXe $(2.8 \mathrm{~cm})$, the most of gamma rays convert in the shallow part of the detector. For example, $40 \%$ of gamma rays convert within $2 \mathrm{~cm}$.

\begin{tabular}{c|c}
\hline \hline Energy resolution [\%] & $2.4 / 1.7$ \\
\hline Position resolution $[\mathrm{mm}]$ & 5 \\
\hline Time resolution $[\mathrm{psec}]$ & 67 \\
\hline Efficiency & 63 \\
\hline
\end{tabular}

Table 1: Performance of the present MEG gamma-ray detector. The left and right numbers of the energy resolution corresponds for events with conversion point shallower than $2 \mathrm{~cm}$ and deeper than that, respectively.

Figure 3 shows the energy response of the gamma-ray detector for $55 \mathrm{MeV}$ monochromatic gamma rays from neutral pion decays. The spectrum shape of the right part is determined by the detector resolution for constant energy deposits, while the long tail of the left part is due to the energy leaks from the sensitive volume and the energy losses of gamma rays before entering the LXe for example in PMTs.

\subsection{Upgrade concept}

For improving the uniformity of the energy response, it is important to use photo-sensors which have a uniform response over the sensitive area and to decrease the fraction of the dead area on the inner face of the gamma-ray detector. For the purpose, silicon photomultipliers (SiPM) are good candidates for replacing the PMTs on the inner face. However there are issues to use SiPMs instead of PMTs in LXe detectors.

- There are no commercially available SiPMs sensitive to VUV photons. 


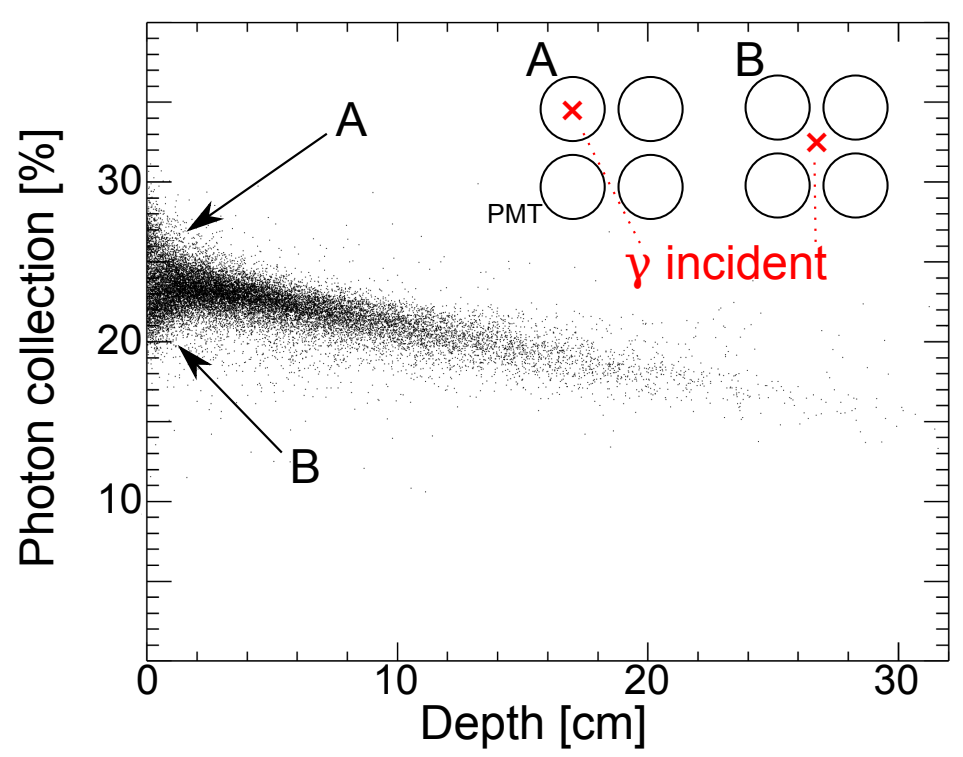

Figure 2: The photon collection efficiency in various positions in the detector as a function of the depth. In the shallow part, the collection efficiency depends on the relative position to the PMT array. In front of a PMT (position-A) the efficiency is high, while it is low between PMTs (position-B).

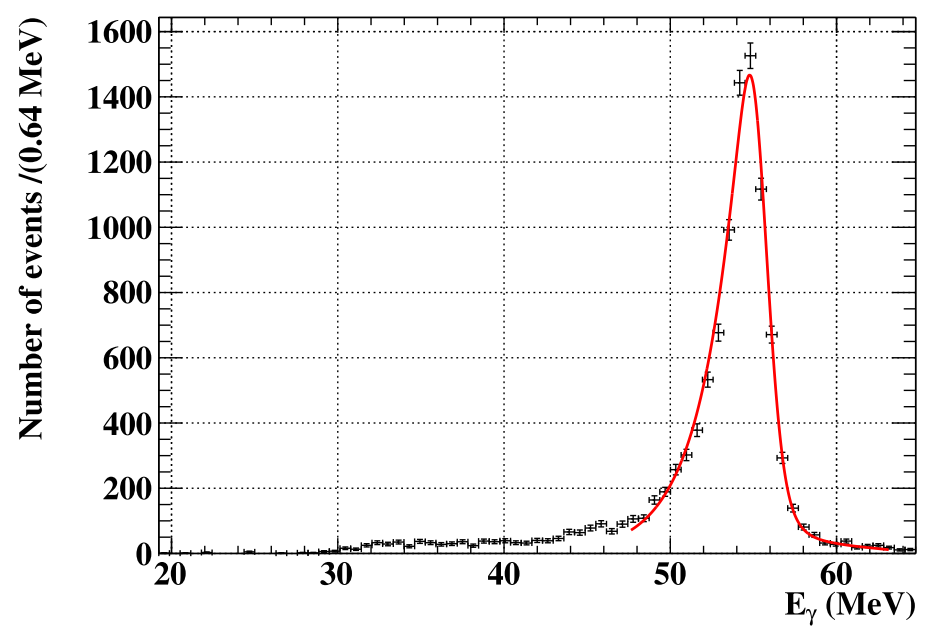

Figure 3: Energy response of the gamma-ray detector for $55 \mathrm{MeV}$ monochromatic gamma rays. 
- With normal size of SiPMs (e.g. $3 \times 3 \mathrm{~mm}^{2}$ ), the number of channels to cover the inner face without making too large dead area is too large. Because we record waveform of all the channels, the cost for read-out electronics and the data size would be too high. The limited available space to put cables in the cryostat and the incoming-heat through many cables could be also issues.

To overcome the issues, we need to develop a new type of SiPMs, which has about $15 \%$ of photon detection efficiency (PDE), a good gain uniformity and the sensitive area size of $12 \times 12$ $\mathrm{mm}^{2}$.

In addition to replacing the photo-sensors, the width of the inner face will be increased to decrease the energy leaks from the lateral faces, and the direction of lateral PMTs will be changed for more uniform energy response (Fig. 4).

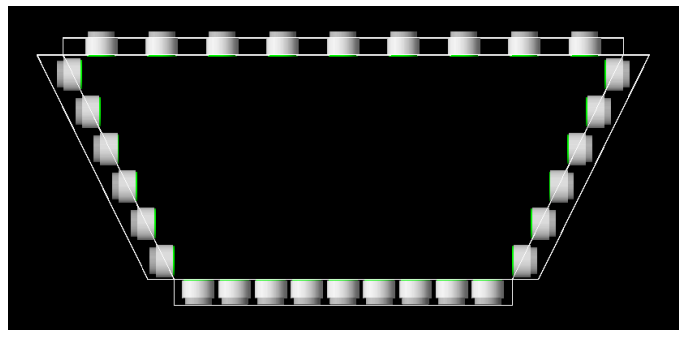

(a) Present detector

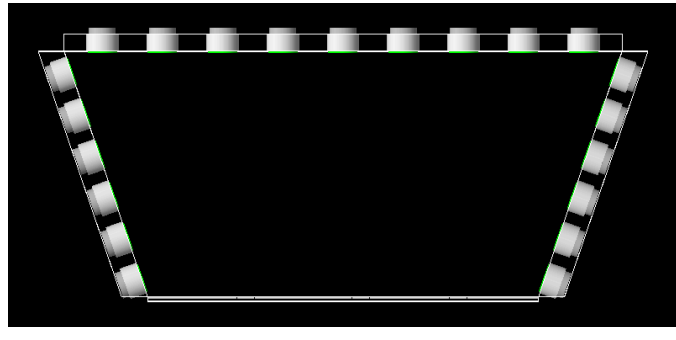

(b) Upgraded detector

Figure 4: Cross section view of the gamma-ray detector. The photo-sensors on the inner face (bottom in the figure) will be replaced with SiPMs; the inner face will be widened; the direction of the lateral PMTs will be changed.

The performance of the upgraded detector is estimated with using Monte Carlo simulation with assuming photo-sensors with the size of $15 \times 15 \mathrm{~mm}^{2}$ and the sensitive area of $12 \times 12 \mathrm{~mm}^{2}$. To cover the inner face, about 4000 photo-sensors are needed. Figure 5 shows the energy response for the signal gamma rays. The sigma of the right part is improved from 2.4/1.7\% to $1.1 / 1.0 \%$, where each number is the resolution in the shallow part (depth $<2 \mathrm{~cm}$ ) and in the deep part (depth $\geq 2 \mathrm{~cm}$ ), respectively. The average position resolution will be improved from $5 \mathrm{~mm}$ to 2.6 (horizontal) or 2.2 (vertical) $\mathrm{mm}$. The detection efficiency will be higher by $10 \%$ because of the smaller thickness of SiPMs compared to PMTs.

\subsection{VUV-sensitive MPPC development and tests}

We developed a new type of multi-pixel photon counter (MPPC, a product name of SiPMs) with Hamamatsu Photonics. Figure 6 shows a schematic view and a photograph of the new MPPC (model S10943-3186(X)). The sensitive part is subdivided into four segments, and each segment has its anode and cathode pins so that the segments can be read individually or can be connected in different schemes. The sensitive part is specially designed and optimized to detect VUV photons in LXe. A protection layer exists in normal MPPCs is removed because VUV photons can be easily absorbed in the layer before entering the sensitive layer. The optical property of the surface is optimized. As the protection layer is removed, the device is too fragile to handle. For the 


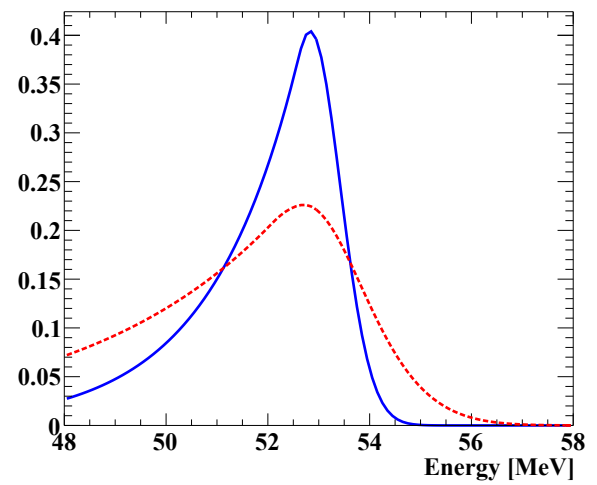

(a) Shallow events

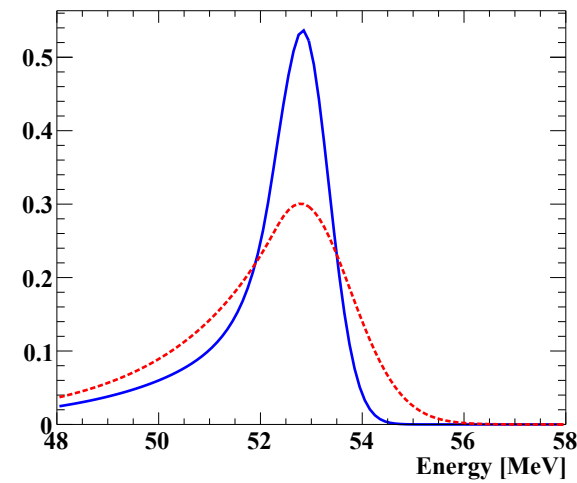

(b) Deep events

Figure 5: Energy response of the present detector (dashed line) and upgraded detector (solid line). The shallow and deep events are separated at the depth of $2 \mathrm{~cm}$.

protection during the assembly and the operation of the detector, a thin quartz window is attached on the ceramic base. The space between the window and the silicon segments is open so that the part can be evacuated and LXe can enter. We made three types of MPPCs with different gap sizes, $0.5,1.0$ and $1.5 \mathrm{~mm}$, to check if conductive dusts, which might float in LXe, could cause a short circuit between segments. The pixel pitch is $50 \mu \mathrm{m}$; the total number of pixels is $47-56$ thousands depending on the width of the gap. The quenching resisters are made of metal so the temperature coefficient is much smaller than conventional polysilicon.

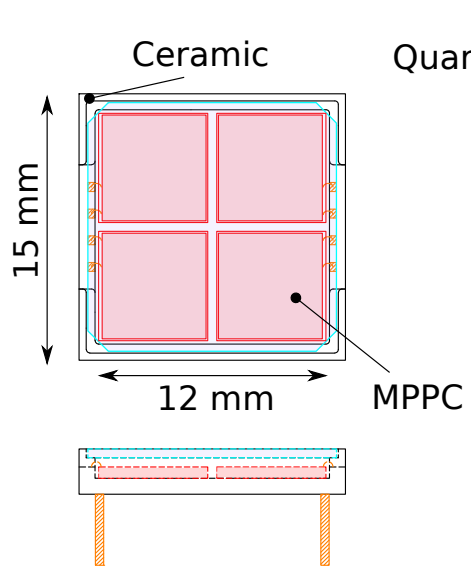

(a)

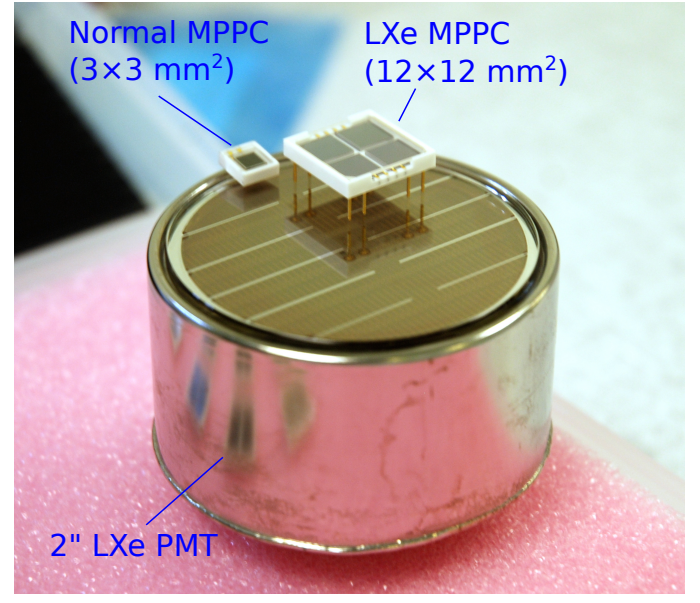

(b)

Figure 6: (a) Schematic view and (2) Photograph of the new MPPC. In (b), a normal MPPC $\left(3 \times 3 \mathrm{~mm}^{2}\right)$ and a 2-inch PMT used in the MEG LXe detector are shown for comparison.

As the size of MPPCs increases, the large sensor capacitance makes the fall time of the signal pulses longer. It makes the signal-to-noise ratio worse. The long tail can increase the probability of pileup events in a high-rate environment like the MEG experiment. For example, the fall-time 
is about $200 \mathrm{nsec}$ when we use a MPPC with the sensor size of $12 \times 12 \mathrm{~mm}^{2}$. Connecting the four segments in parallel is equivalent to use a non-segmented MPPC; while by connecting the segments in series, the sensor capacitance can be much reduced. The signal fall time was measured with connecting the segments in three ways: (1) all in parallel, (2) series connection of two parallel connected segment pairs, (3) all in series. The pulse-height does little depend on the connection scheme. The measured signal fall time is 200,50 and $30 \mathrm{nsec}$ with the three connection schemes, respectively. The signal pulses of the new MPPCs can be narrow enough by connecting the segments in series. The different electric potential of the segments can be a problem when some conductive dusts make a short between the segments. For avoiding the problem, we are going to use "hybrid" connection (Fig. 7). With the hybrid connection the signal pulses go through the capacitors between the segments and the circuit works like a series connection, while the bias line is cut by the capacitors and the circuit works like a parallel connection. By using the circuit, the signal pulses become narrow while the electric potential of the segments is common.

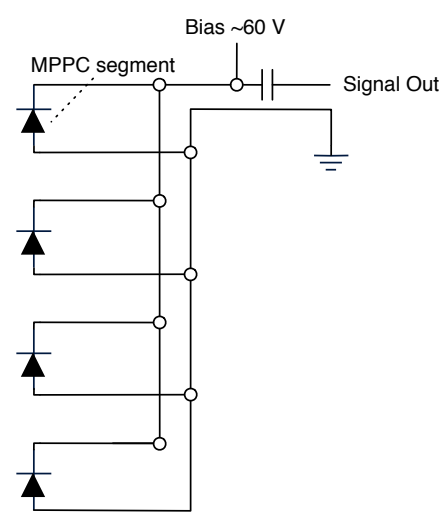

(a) Parallel

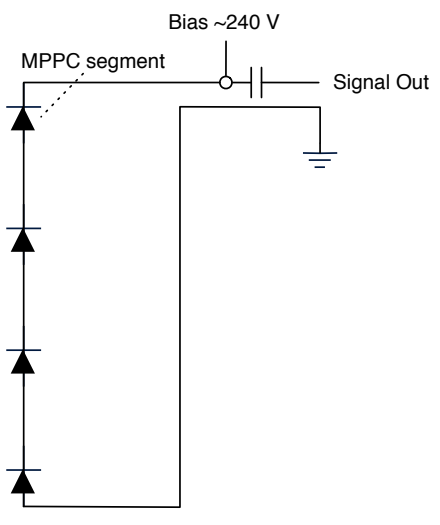

(b) Series

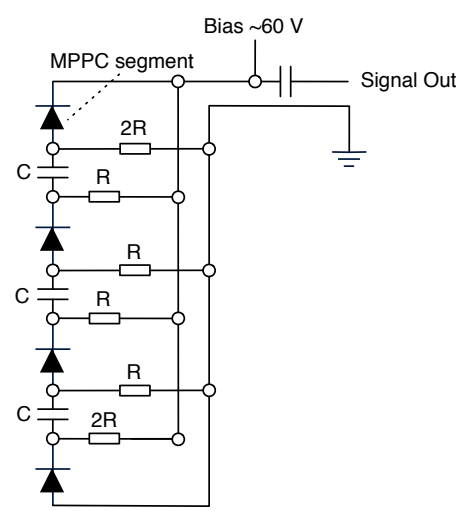

(c) Hybrid

Figure 7: Connection schemes of four MPPC segments. The typical capacitance $\mathrm{C}$ and resistance $\mathrm{R}$ in the hybrid connection is $10 \mathrm{nF}$ and $10 \mathrm{k} \Omega$, respectively.

We tested prototype samples of the new MPPC in a small LXe chamber. About two liters of xenon is liquefied in the chamber and properties of the MPPCs are measured. To measure the PDE, an ${ }^{241} \mathrm{Am}$ source, which emits $5.5 \mathrm{MeV}$ alpha rays, is put in the LXe and the number of observed photoelectrons is compared with the expectation from the geometrical acceptance. The measured properties are summarized in Table 2. We confirmed that the single photoelectron peak of the charge-histogram is visible, which means the gain of pixels over the large sensitive area is uniform.

The first mass production of 600 MPPCs was done in March 2014. We are going to measure properties of every segment individually in room temperature to know the distribution of them. The tests will be done also in LXe with using a large cryostat where $\sim 100$ liters of xenon can be liquefied. 


\begin{tabular}{c|c}
\hline \hline Gain & $3 \times 10^{5}$ \\
\hline PDE for LXe scintillation & $15 \pm 3 \%$ \\
\hline Dark rate & $\sim 150 \mathrm{~Hz}$ \\
\hline Crosstalk probability & $35 \%$ \\
\hline Afterpulse probability & $10 \%$ \\
\hline Signal fall time & $30 \mathrm{nsec}$ \\
\hline
\end{tabular}

Table 2: Properties of the new type of MPPC. The measurement was done in LXe. The temperature is $\sim 165 \mathrm{~K}$. The four segments were connected in series. The nominal operation voltage of $2.5 \mathrm{~V}$ per segment was applied. The effects of crosstalk and afterpulse are subtracted in the evaluation of the PDE.

\section{Conclusion and prospects}

For improving the sensitivity of the MEG experiment, the detector is being upgraded. The LXe gamma detector will also be upgraded with modifying the profile of the sensitive part and with replacing the 216 2-inch PMTs on the inner face with $\sim 4000$ MPPCs. The energy and position resolutions are expected to be much improved especially in the shallow part. We developed new VUV sensitive MPPCs with Hamamatsu Photonics. Properties of test-samples of the MPPC were measured in LXe. The gain and PDE are large enough. The signal-pulses can be narrow enough by connecting the segments in series or with using "hybrid" connection. We are going to test 600 sensors produced at the first mass-production. After the test, the remaining sensors will be produced. The final detector is supposed to be build by year 2016 to start the MEG II experiment with a ten times better signal sensitivity than the MEG experiment.

\section{References}

[1] J. Adam et al. (MEG Collaboration), New Constraint on the Existence of the $\mu^{+} \rightarrow e^{+} \gamma$ Decay, Phys. Rev. Lett. 110 (2013) 201801. 